\title{
Desafíos de las Estrategias Pedagógicas Integradas (EPI) en la formación de profesores y profesoras de Educación Física en Chile Challenges of integrated learning Strategies (ILS) in a Physical Education teaching training program in Chile
}

*Braulio Ademir Navarro Aburto, **Sebastián Peña Trocoso, ***, ****Juan Carlos Beltrán, ***José Luis Gálvez Nieto, *****Adolfo Guzmán Muñoz, ******Elizabeth Flores Ferro, *Paola Fuentes Merino *Universidad Autónoma de Chile (Chile), **Universidad Austral de Chile (Chile), ***Universidad de la Frontera deTemuco (Chile), ****Universidad Mayor deTemuco (Chile), *****Universidad SEK (Chile), ******Universidad Bernardo O’Higgins (Chile)

Resumen. Los esfuerzos que desarrolla el contexto universitario en Chile en la formación de profesores de educación física vinculados a las Estrategias Pedagógicas Integrada (EPI) son plausibles, pero, se plantea que toda propuesta pedagógica para alcanzar el rango de calidad necesita ser evaluada, lo que permite cumplir con los objetivos que generaron su creación. El objetivo del estudio fue identificar los obstáculos y desafíos que profesores y estudiantes de la carrera de pedagogía en educación física de una universidad en Chile, manifiestan de la EPI. La metodología utilizada estuvo dada por el enfoque cualitativo. La principal técnica de recolección de datos se realizó a través de la entrevista en profundidad. Para la reducción de los datos cualitativos se utilizó el software ATLAS Ti. 7.5. Los participantes de este estudio fueron diez estudiantes y diez profesores de la carrera de Pedagogía Educación Física en Temuco, Chile. Los principales resultados manifiestan, falta de articulación docente y el trabajo colaborativo entre el estudiantado que permite articular el trabajo en el proceso de la EPI. Como conclusión se manifiesta que es necesario dejar de lado la mirada técnica del proceso que lleva a enfocar la EPI solo en los resultados, haciendo énfasis en el proceso EPI que enriquece la formación docente en la praxis educativa.

Palabras claves: pedagogía, educación, formación inicial, estrategias pedagógicas integradas.

\begin{abstract}
Efforts developed in the university context in Chile regarding physical education (PE) teaching training linked to integrated learning strategies (ILS) are plausible. However, it is stated that all proposed pedagogical approaches, to reach a high standard quality, require assessment to allow them to accomplish the objectives that prompted their creation. The aim of this study was to identify the obstacles and challenges that professors and students have encountered related to ILS in a PE training program from a Chilean university. The methodology employed was based on the qualitative approach and the main data collection tool was an in-depth interview. ATLAS ti. 7.5 software was used to reduce and define the qualitative data gathered. The participants of this research were 10 students and 10 professors from a PE teaching training program in Temuco, Chile. Results show the main obstacles and challenges for ILS are associated with a lack of structured teaching approach and of collaborative work among the student body, which allow efficient management of the ILS working process. As a conclusion, it is stated the need to put aside the technical approach focusing ILS only on results, to give emphasis instead to the ILS process, which enriches teaching training in the educational praxis.
\end{abstract}

Keywords: pedagogy, education, initial teacher training, integrated pedagogical strategies.

\section{Antecedentes}

La investigación se sitúa en el contexto universitario chileno y nace de la experiencia de un grupo de profesionales vinculados a la ejecución y acompañamiento estudiantil denominado Estrategia Pedagógica Integrada (EPI) la que consiste en el apoyo interasignaturas, la integración de actividades formativas a través de acuerdos metodológicos y evaluativos que se derivan del análisis del diagnóstico de los estudiantes, de los resultados

Fecha recepción: 13-05-20. Fecha de aceptación: 14-05-21

Braulio Ademir Navarro Aburto

braulio.navarro@ubo.cl de aprendizaje comprometidos en las asignaturas, el balance de carga de trabajo autónomo y de otras necesidades emergentes durante el semestre para la atención de los estudiantes y que se van desarrollando a través de reuniones mensuales en las que los profesores de las asignaturas del mismo nivel conforman una comunidad académica para la planificación de la EPI, su aplicación, seguimiento y análisis de resultado (UA, 2017).

El objeto de estudio lo constituye la estrategia pedagógica integrada (en adelante EPI), Tolozano, Lara y Illesca (2015) plantean que en las instituciones de Educación Superior constituyen una actividad permanente que requiere de una creciente participación de los docentes y que permite satisfacer la necesidad de centrar 
la atención de la institución y de sus profesores en los estudiantes a lo largo de su formación académica.

Los programas vinculados a las EPI, son utilizados como una estrategia de prevención para aumentar los apoyos sociales y habilidades de los estudiantes de Educación Superior. Incluye instrucción, apoyo emocional, ayuda financiera, colocación laboral, proyectos de investigación, entre otros. En la academia, se relaciona con mayores tasas de retención, graduación y para elevar las percepciones positivas de los estudiantes sobre la institución educativa (Grant-Vallone y Ensher, 2000).

Para Cardozo-Ortiz (2011) la EPI, poco a poco, se ha convertido en un tema de interés para las instituciones educativas superiores, dada la necesidad de brindar a los(as) estudiantes nuevas oportunidades de aprendizaje, en las cuales ellos sean los principales actores.

Calle y Saavedra (2009) plantean que:

La EPI vinculada a la tutoría es indispensable para promover la investigación formativa, por cuanto se hace necesario que el estudiante, previamente orientado por el tutor, inicie sus búsquedas y consultas relacionadas con el tema propuesto; por tanto, el trabajo de asesoría no siempre trata de aportar información o sugerir fuentes para buscarla, aclarar conceptos o procedimientos y recomendar acciones de ejercitación y verificación de habilidades y conocimientos. Suele ser una tarea tan compleja, que requiere de la comunidad educativa de paciencia y habilidad para detectar las posibles causas por las cuales el estudiante no logra acercarse a los conocimientos propuestos, o trabajar un tema, concepto o proceso particular (p.319).

La evidencia empírica manifiesta un creciente interés en los efectos y alcances de las estrategias pedagógicas integradas vinculadas a tutoría a nivel universitario (Andreucci-Annunziata y Curiche, 2017; Cardozo-Ortiz, 2011; Calle y Saavedra, 2009; De la Cruz, Chehaybar y Abreu, 2011; Hernández, C. Jiménez, M., Guadarrama, E., y Rivera, A. (2016); Forestello, 2014; Molina, 2012; Lobato y Guerra, 2014; Mayer y Cerezo, 2016; Tolozano, Lara y Illesca, 2015; Torrado, Manrique-Hernández y Ayala, 2015), pero, adolece de una mirada crítica y reflexiva en relación a su implementación, es decir, visualizar desde la realidad vívida de los profesores, estudiantes, y encargados del programa, el nivel de satisfacción y los obstáculos y desafíos de las EPI.

Para Ehrich, Hansford yTennet, (2003, citado en De la Cruz, et al. 2011) los principales obstáculos que presentan las EPI asociadas a tutorías académicas está dado por poca disponibilidad en cuanto a tiempo por parte de los docentes, problemas interpersonales, incompatibi- lidad de caracteres, falta de compromiso y las diferentes expectativas entre la comunidad educativa y el estudiantado.

En relación a los desafíos planteados, se puede inferir en correspondencia a lo esbozado por la investigación, que la EPI, debe propiciar los resultados de aprendizaje mediante el uso de métodos de enseñanza constructivistas y socio-constructivistas, en donde debe existir una participación activa de todos los profesores(as) de las asignaturas, dado que una verdadera labor de acompañamiento requiere de su orientación y compromiso para lograr la verdadera mediación pedagógica para favorecer los resultados de aprendizaje y la consecución del perfil de egreso comprometido $(\mathrm{Ca}-$ lle y Saavedra, 2009; Cardozo-Ortiz, 2011; Forestello, 2014; Molina, 2012; Tolozano, Lara y Illesca, 2015; Torrado, Mayer y Cerezo, 2016).

De acuerdo con lo anterior, la formación universitaria en Chile y en el contexto internacional de profesores(as) de Educación Física busca aportar al desarrollo académico integral de los estudiantes, implementando mecanismos de apoyo de alta calidad en el ámbito académico y socioemocional, que buscan fortalecer en estos las competencias genéricas y específicas necesarias para su correcta adaptación y desenvolvimiento en el contexto universitario (Carreño et. al, 2019; Flores et. al, 2020; Carreiro, González y González, 2016).

Para la formación universitaria en Chile de profesores(as) de Educación Física toda iniciativa educativa debe alcanzar un rango de calidad y transparencia, que necesita ser analizada, lo que permitirá mejorar su aplicación, pero, por sobre todo cumplir con los objetivos y lineamientos que generaron su creación, por lo cual, a través de una aproximación metodológica cualitativa se plantea como objetivo: identificar los obstáculos y desafíos que profesores(as) y estudiantes manifiestan sobre la EPI en la carrera de Pedagogía en Educación Física de una universidad en Chile:

\section{Metodología}

La investigación se genera desde el paradigma interpretativo y el enfoque cualitativo pues busca comprender e interpretar las dinámicas sociales desde el ser situado históricamente (Flick, 2009). En el estudio participaron 10 profesores que conforman el cuerpo docente de la carrera participante en la EPI y 10 estudiantes de la carrera de Pedagogía en Educación Física de una universidad en Chile, que fueron parte de la EPI 
en el año 2018, cuyo criterio de inclusión fue participación voluntaria en el estudio. Es importante señalar que, para cumplir con los criterios éticos propuestos, se generó el consentimiento informado y la autorización del comité de ética al ser esta una investigación patrocinada por la Universidad (Proyecto DIDUA /2018). La técnica de recolección de datos fue la entrevista en profundidad, la cual permitió captar la riqueza de los significados emanados de los participantes para comprender el fenómeno de investigación, ya que, la información acerca de los obstáculos y desafíos que profesores y estudiantes manifiestan de las estrategias pedagógicas integradas es escasa. Esta técnica, permite asegurar la elaboración de preguntas que todos van a responder, por la facilidad en la administración y evaluación, el entrevistador no requiere un entrenamiento profundo, además son entrevistas más pequeñas, por lo que los datos son más fáciles de manejar (Escribano, 2008).

\section{Técnica de recolección de datos}

se generaron pautas de entrevistas semiestructuradas que emergen a priori de los objetivos específicos de la investigación (, las entrevistas fueron sometidas a juicio de expertos que permitió corregir las pregunta cuestionadas por los especialistas vinculados al tema, luego se genera el pilotaje del instrumento para a posteriori ser aplicada de manera formal en los sujetos de estudio. Las entrevistas fueron grabadas en sistema de audio MP3 y transcritas a documento Word.

\section{Plan de Análisis}

Para identificar los obstáculos y desafíos que profesores y estudiantes de la carrera de Pedagogía en Educación Física de una universidad en Chile manifiestan de la EPI desde el método cualitativo, es necesario analizar las respuestas de los(as) entrevistados, para que permita leer e interpretar sus relatos (Ruiz, 2003). Se organizó la información a través de codificación axial utilizando software ATLAS.ti 7.5. Este software agiliza de forma considerable las actividades implicadas en el proceso de análisis de las entrevistas, como la segmentación del texto en citas, el proceso mismo de codificación, la escritura de memos y anotaciones, y la realización de vínculos entre citas, memos y códigos. Para el proceso de codificación de los datos se incorporaron elementos de la teoría fundamentada, como la codificación abierta y axial. La codificación abierta, permite descubrir, nombrar y desarrollar los conceptos, se debe abrir el texto y exponer los pensamientos, ideas y significa- dos contenidos en él (Staruss y Corbin, 2002), y des esta forma, levantar categorías y subcategorías. Consecutivamente, se aborda la codificación axial, este proceso de análisis se entiende, como depurar y diferenciar las categorías derivadas de la codificación abierta. Las categorías axiales se enriquecen por su ajuste con el mayor número de pasajes posible, para luego elaborar las relaciones entre categorías (Flick, 2007) y subcategorías. Los procesos de codificación se realizaron, mediante la utilización del Método Comparativo Constante (MCC), «este procedimiento se convierte en un método de comparación constante cuando los intérpretes cuidan de comparar los códigos una y otra vez con los códigos y las clasificaciones obtenidas» (Flick, 2007, p. 248). Posteriormente, se presentan los resultados, en este apartado se describen las categorías y se presentan los resultados por subcategorías. Finalmente, se discuten los resultados por categoría, a la luz de la teoría.

\section{Resultados y discusión}

La presentación de los resultados obtenidos se realizó mediante la visualización de figuras organizadas en dos grandes categorías: a) obstáculos; b) desafíos y sus respectivas sub-categorías las cuales se describen y contrastan con los respaldos bibliográficos correspondientes.

\section{Presentación de redes esquemas/matrices}

A continuación, se presenta la figura 1 vinculada al objetivo identificar los obstáculos que el profesorado y estudiantado de la carrera de Pedagogía en Educación Física de una universidad en Chile manifiestan de la EPI.

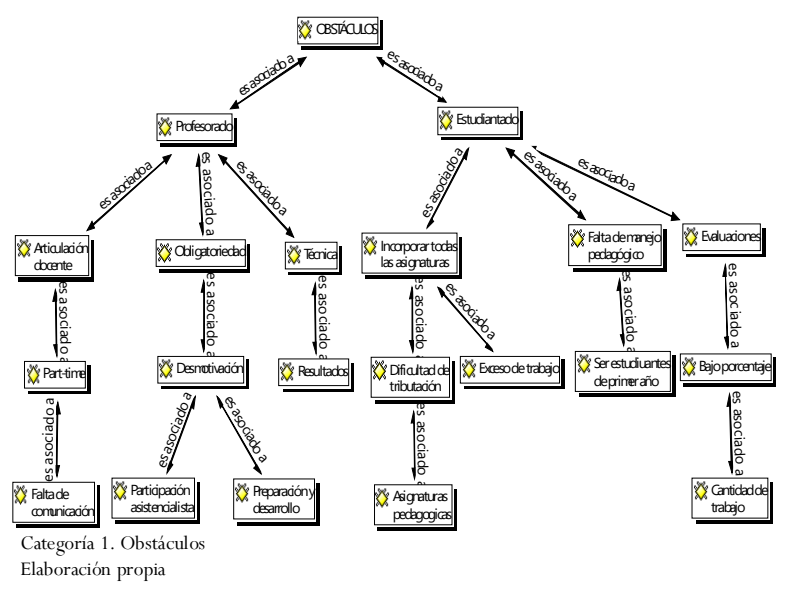

\section{Categoría 1. Obstáculos}

Se presentan las categorías profesorado y alumnado de las cuales emergen las siguientes subcategorías: articulación docente, part-time, falta de co- 
municación, obligatoriedad, desmotivación, participación asistencialista, preparación y desarrollo, técnica, resultados, incorporar todas las asignaturas, dificultad en la tributación, asignaturas pedagógicas, exceso de trabajo; falta de manejo pedagógico, ser estudiante de primer año, evaluaciones, bajo porcentaje, cantidad de trabajo.

De acuerdo al relato y análisis de la entrevista en profundidad vinculado a la categoría profesorado y la subcategoría articulación docente, el profesorado plantea que uno de los principales obstáculos se vincula a la falta de articulación docente generada por la presencia de un número de profesores que no poseen jornada completa de trabajo y que constituyen el grupo de docentes tipo part-time, lo anterior genera que la articulación docente no se formalice, por lo cual, la estrategia didáctica que es la esencia del proceso se desvirtúe producto de falta de comunicación. Esto lo respalda la siguiente textualidad: «el compromiso de algunos profesores que por lo general son los part-time, no tienen tanto compromiso con la EPI, porque si bien le pagan una hora al mes, mayor rédito no tienen».

En relación con los obstáculos y a la subcategoría obligatoriedad, el profesorado manifiesta que la universidad plantea el proceso de la EPI como un proceso pedagógico impuesto y rígido que genera desmotivación del cuerpo académico y, por ende, se transforma en un asunto de intervalos unidireccionales que descontextualiza su objetivo. Respalda lo anterior el siguiente relato: «a la larga esto va a ser contraproducente, en vez de que ojalá fuera al revés, que ojalá entre nosotros viéramos estrategias colaborativas de forma más natural y que no tan obligadas».

Respecto del relato y análisis de las entrevistas asociado a los obstáculos se identificó la subcategoría participación asistencialista, el profesorado manifiesta que la universidad en su afán inclusivo funcional genera una serie de estrategias que quitan la autonomía al estudiantado, lo anterior genera problemas en preparación y desarrollo de los procesos pedagógicos pues se asume que para generar éxito en la EPI se debe entregar y asesorar todos los elementos al alumnado, dejando de lado el proceso de descubrimiento autónomo de la didáctica educativa. Lo anterior genera un énfasis en la técnica y produce que todo el proceso se situé en los resultados, emergiendo la racionalidad técnica instrumental como centro del proceso pedagógico. Esto lo respalda la siguiente textualidad: «de las cosas que yo he escuchado sobre todo de los docentes que llevan más años, las personas más mayores consideran que este tipo de estrategias son muy asistencialistas».
De acuerdo con el relato y análisis de la entrevista en profundidad vinculado al alumnado de Pedagogía en Educación Física y la subcategoría incorporar todas las asignaturas, el estudiantado manifiesta que la lógica de incorporar todas las materias en el proceso EPI genera un gran obstáculo debido a que por sus objetivos y característica es muy difícil que converjan, lo cual, origina dificultad en la tributación. Lo anterior se vincula a que desde la disciplina Educación Física y desde las propuestas prácticas de las estrategias pedagógicas integradas la articulación de las asignaturas pedagógicas se hace complejo. Esto se manifiesta en la siguiente declaración «algunas asignaturas costaba integrarlas con las demás, no era fácil tributarlas».

Además, el alumnado con relación a los obstáculos y a la subcategoría exceso de trabajo, los estudiantes manifiestan que debido a que la EPI se realiza mediante el trabajo articulado de las diversas asignaturas, este proceso se transforma en una cátedra más, lo cual, incrementa su trabajo académico, sumado a la falta de manejo pedagógico por ser estudiante de primer año. Lo anterior lo respalda la siguiente textualidad: «el poco conocimiento que teníamos al principio por venir ingresando a la universidad, ya que teníamos algo, pero no lo necesario»

Por último, el estudiantado declara que uno de los obstáculos más evidentes se vincula a el exceso de trabajo que generan evaluaciones con bajo porcentaje que no se relacionan con la gran cantidad de trabajo que conlleva la ejecución de la EPI. Lo anterior se expresa en la siguiente afirmación «la EPI era mucho trabajo y la evaluación muy baja en el porcentaje».

Respecto de los obstáculos para la implementación de la EPI en la formación de profesores de Educación Física se evidencia de parte de los(as) profesoras y los(as) estudiantes que la EPI carece de articulación docente como centro del proceso pedagógico y como el más importante acto didáctico del proceso de enseñanza aprendizaje. Esto genera falta de comunicación sumando a la obligatoriedad de participación, obteniendo como producto la desmotivación y asistencialismo.

De acuerdo a lo anterior, los resultados no se acercan a lo planteado por Cardozo-Ortiz (2011) quién señala que la EPI, poco a poco, se ha convertido en un tema de interés para las instituciones educativas superiores, dada la necesidad de brindar a los(as) estudiantes nuevas oportunidades de aprendizaje, en las cuales ellos sean los principales actores. De acuerdo a lo planteado, la universidad obliga a los profesores a trabajar en EPI como un proceso pedagógico impuesto y rígido que 
genera desmotivación del cuerpo académico y, por ende, la EPI se transforma en un asunto de intervalos unidireccionales que descontextualiza su objetivo.

Para Driscoll (2005), las estrategias de enseñanza centradas en el estudiante deben responder a un aprendizaje situado, dado que la construcción del conocimiento es concebida como una práctica en la experiencia, por lo que aprender implica involucrarse en una comunidad de práctica. El aprendizaje situado depende de las actividades, del conocimiento y de la cultura que coexisten en un contexto auténtico donde ocurre una interacción social de los miembros de la comunidad (Páramo, Hederich, López, Sanabria, y Camargo, 2015)

Si bien el principal objetivo de la EPI es lograr un aprendizaje stuado desde estrategias didácticas centradas en el alumno(a) la instrumentalización de un proceso impuesto desencadena confusión y problemáticas en la implementación.

Por último, los principales obstáculos presentes en los hallazgos se relacionan con los planteado por Ehrich, Hansford y Tennet, (2003, citado en De la Cruz, et al), en relación a que las principales dificultades que presentan las estrategias pedagógicas integradas asociadas a tutorías académicas está dado por poca disponibilidad en cuanto a tiempo por parte de la comunidad educativa, problemas interpersonales, incompatibilidad de caracteres, falta de compromiso y expectativas diferentes entre la comunidad educativa y el estudiantado.

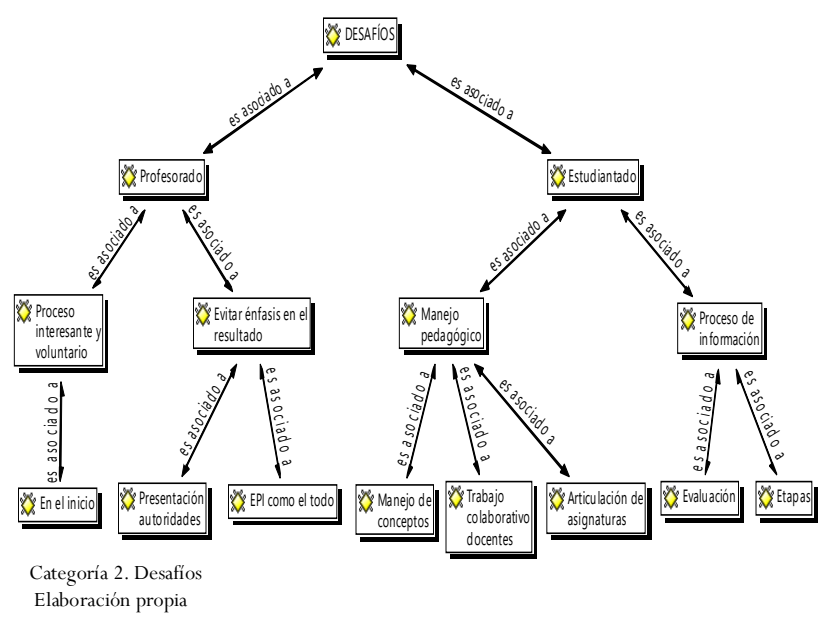

A continuación, se presenta la figura 2 vinculada al objetivo identificar los desafíos que el profesorado y estudiantado de la carrera de pedagogía en educación física de una universidad en Chile manifiestan de la estrategia pedagógica integrada (EPI)

\section{Categoría 2. Desafíos}

Se presentan las categorías profesorado y estu- diantado de las cuales emergen las siguientes subcategorías: proceso interesante y voluntario, en el inicio, evitar hacer énfasis en el resultado, presentación a las autoridades, EPI como el todo, manejo pedagógico, manejo de conceptos, trabajo colaborativo con docentes, articulación de asignaturas, proceso de información, evaluación, etapas.

De acuerdo con el relato y análisis de la entrevista en profundidad vinculado a la categoría profesorado y la subcategoría proceso interesante y voluntario, los(as) entrevistadas manifiestan que los principales desafíos se asocian a que el proceso de la EPI resulta interesante, pero no en su fase de construcción en el inicio, sino más bien en su fase de aplicación. Por otra parte, se asume que es indispensable la participación docente voluntaria en el trabajo de la EPI, evitando que esta sea impuesta como parte de los productos académicos, por los cuales, los docentes son evaluados. Lo anterior lo respalda la siguiente textualidad: «han encontrado el proceso EPI interesante al final, cuando ojalá lo hubiesen visto desde el principio»

Además, el profesorado en relación con los desafíos y la subcategoría evitar hacer énfasis en el resultado, declara que la estrategia pedagógica integrada es parte del programa de comunidad académica, pero, cuando esta es llevada y situada en una presentación a las autoridades, pierde su sentido pedagógico de proceso, transformando la EPI en el todo, es decir, en un producto de intercambio entre autoridades y docentes. Lo anterior, es declarado en la siguiente afirmación «se enfoca la EPI como lo más importante, como la guinda de la torta del proceso comunidad académica y de todos los mecanismos que se debiesen actuar, en el funcionamiento hay varias cosas que se hacen. Lo que más luce es la EPI, es decir el resultado» (E3:2).

De acuerdo con el relato y análisis de la entrevista en profundidad vinculado a la subcategoría manejo pedagógico, los estudiantes manifiestan que el principal desafío de la EPI se vincula a poseer mayor experiencia formativa docente que les permita un mejor manejo de conceptos. Lo anterior les permitirá involucrase desde el conocimiento teórico y práctico de la disciplina y fortalecerá su proceso de formación. Lo ya señalado se visualiza en el siguiente relato: «más manejo teórico en la práctica de la EPI, salió súper bien, pero, había algunos vacíos teóricos, también porque éramos de primer año».

Por último, los estudiantes declaran que es fundamental fortalecer el trabajo colaborativo con docentes, robustecer la articulación de asignaturas, mejorar los procesos de información, en cuanto a la evaluación y etapas del 
proceso de desarrollo de las estrategias pedagógicas integradas. Lo anterior lo respalda la siguiente textualidad: «es necesario mejorar la articulación de asignatura y mejorar los procesos de información, y subir los porcentajes de las evaluaciones».

Respecto de los desafíos para la implementación de la EPI en la formación de profesores de Educación Física se evidencia de parte de profesores y estudiantes que el estudio se vincula a lo que plantean diversos autores (Cardozo-Ortiz, 2011; Calle y Saavedra, 2009; Forestello, 2014; Tolozano, Lara y Illesca, 2015; Molina, 2012; Mayer y Cerezo, 2016; Torrado, ManriqueHernández y Ayala, 2015) con relación al manejo pedagógico que deben poseer los estudiantes, trabajo colaborativo con docentes que permita mejorar la articulación de asignaturas, optimizando el proceso de información, evaluación y etapas de la EPI.

Además, las comunidades educativas vinculadas a la EPI deben fortalecer las características pedagógicas y metodológicas para los alumnos y alumnas, generar instancias para una relación de comunidad educativa, es necesaria para mayor difusión dentro de la comunidad educativa de las EPI, y fortalecer la participación activa de los profesores(as) de las asignaturas, dado que una verdadera labor de acompañamiento requiere de su orientación y compromiso para lograr una apropiada mediación y articulación pedagógica desde una perspectiva situada.

\section{Conclusiones}

Las conclusiones del estudio se presentan de acuerdo al objetivo de investigación: identificar los obstáculos y desafíos que profesores y estudiantes de la carrera de Pedagogía en Educación Física de una universidad en Chile, manifiestan de la EPI.

Con el fin de operacionalizar este propósito se presentan las categorías de análisis en torno a las cuales se muestran las conclusiones.

En relación con la categoría de obstáculos planteados por el profesorado y estudiantado que están en proceso de formación y han sido parte de la EPI, estos se vinculan principalmente a problemas en la articulación docente generadas por profesores y profesoras que trabajan en la universidad por horas (part-time), sin poseer una jornada que asegure su presencia permanente en la universidad. Lo anterior tiene como consecuencia problemas en la comunicación, lo que impacta en el desarrollo y preparación de la EPI. Además, la EPI busca la tributación de todas las asignaturas en un trabajo colegiado lo que genera problemática por exceso de trabajo para los estudiantes y dificultad en articular asignaturas de la línea pedagógica que no se acercan a las cátedras de la especialidad.

En relación con la categoría desafíos planteados por el profesorado y estudiantado que están en proceso de formación y han sido parte de la EPI, estos desafíos se vinculan a evitar hacer énfasis en los resultados de la misma, más que en el proceso. Se asume que la EPI es una instancia trasformadora crítica, por lo cual, centrar todo un proceso en los resultados de una reunión ante autoridades universitarias genera una mirada técnica racional de un proceso critico transformador. Por último, se plantea como desafío mejorar el marketing de la EPI, es decir, canalizar la información a los estudiantes a través de medios que sean familiares y de fácil acceso para la comunidad universitaria, planteando qué es la EPI y cuáles serán sus objetivos de proceso.

Es posible concluir de acuerdo con lo planteado por los sujetos de estudio, que las estrategias pedagógicas integradas son una necesidad a nivel del contexto universitario chileno, más allá de las miradas nomotéticas de productividad que se visualizan. Estos espacios permiten la generación de comunidades de aprendizajes que se acercan a los modelos constructivistas y sociocríticos de enseñanza, permiten al estudiante trabajar con un igual, derribando las estructuras clásicas de poder en donde el profesor se sitúa desde una disposición de dominio que dificulta el aprendizaje, por otra parte, permiten que los alumnos y alumnas desarrollen experiencias ligadas a la docencia, y que en la realidad educativa sitúen esta actividad como fuente de desarrollo profesional.

Por último, es necesario manifestar que el esfuerzo que desarrolla la universidad investigada en EPI es plausible, si bien se presentan diversos obstáculos, los encargados del programa son conscientes de ello y manifiestan que también son desafíos que deben abordar. Por otra parte, someter un programa a evaluación voluntaria a través de estudios financiados por la misma universidad manifiesta un alto sentido crítico y reflexivo que es escaso en la educación de mercado predominante, por tanto, devela que el camino elegido es el correcto e invita a que otras comunidades educativas generen acciones similares.

\section{Referencias}

Aguilar-Salinas, W. Chávez-Valenzuela, G. y de las Fuentes-Lara, M. (2017). Tutorías: Estudio Exploratorio 
sobre la Opinión de los Estudiantes de Tronco Común de Ciencias de la Ingeniería. Formación universitaria, 10, 69-80.

Andreucci-Annunziata, P. y Curiche, A. (2017). Tutorías académicas: desafíos de un programa piloto entre pares en una universidad no selectiva. Revista Latinoamericana de Ciencias Sociales, Niñez y Juventud, 15, 357-371.

Ariza, G. y Ocampo, H. (2005). El acompañamiento tutorial como estrategia de la formación personal y profesional: un estudio basado en la experiencia en una institución de educación superior. Universitas Psychologica, 4, 31-42.

Calle, M. y Saavedra, L. (2009). La tutoría como mediación para el desarrollo autónomo del estudiante. Tabula Rasa, 11, 309-328.

Cardozo-Ortiz, C. (2011). Tutoría entre pares como una estrategia pedagógica universitaria. Educación y Educadores, 14, 309-325.

Carreiro, F. Gonzáles, M. y Gonzáles, M. (2016). Innovación en la formación del profesorado de educación física. Revista Retos. 29, 251-257.

Carreño, J. Díaz, A. López, S. y Martín, J. ¿Qué se investiga en formación docente en educación física y en recreación? Revista Retos. 36, 3-8.

De la Cruz, G. Chehaybar, E. y Abreu, L. (2011 ). Tutoría en educación superior: una revisión analítica de la literatura. Revista de la educación superior, 1(157), 189-209.

Flick, U. (Ed.). (2007). Introducción a la investigación Cualitativa. Madrid, España: Ediciones Morata.

Flores, E. Maureira, F. Silva, A. Muñoz, M. Matheu, A. Navarro, B. Bahamondes, V. Hadweh, M. Hurtado, J. Valdés, M. Aedo, E. Peña, S. Serey, D. Zabala, J. Maureira, J. Brevis, M. y Valero, M. (2020). Percepciones institucionales de los estudiantes de Pedagogía en Educación Física de Chile Institutional. Revista Retos. 38, 312-316.

Forestello, P. (2014). Estudiar y aprender en primer año de la Universidad. Praxis Educativa 18(1), 67-74.

Hernández, C., Jiménez, M., Guadarrama, E. y Rivera, A. (2016). La Percepción de la Motivación y Satisfacción de la Tutoría Recibida en Estudios de Posgrado. Formación Universitaria 9(2), 49-58.

Lobato Fraile, C., y Guerra Bilbao, N. (2014). Las Tutorías universitarias en el contexto europeo. Orientación y sociedad, 14.

Mayer, L. y Cerezo, L. (2016). Tutorías y estipendio mensual: contribuciones a la trayectoria universitaria de jóvenes en situación de vulnerabilidad. Revista
Latinoamericana de Ciencias Sociales, Niñez y Juventud, 14(2), 1421-1433. doi: 10.11600/ $1692715 x .14236251115$

Maturana, H. y Varela, F. (Eds.). (2003). El árbol del conocimiento: las bases biológicas del entendimiento humano. Buenos Aires, Argentina: Grupo Editorial Lumen.

Molina Bernal, I. A. (2012). Estado del arte sobre tutorías. Civilizar Ciencias Sociales y Humanas, 12, 167-176.

Páramo, P. Hederich, C. López, O., Sanabria, L. y Camargo, A. (2015). ¿Dónde ocurre el aprendizaje? Psicogente, 18(34), 320-335.

Ruiz-Bolivar, C. (2012). El Enfoque Multimétodo en la Investigación Social y Educativa: Una Mirada desde el Paradigma de la Complejidad. Revista de Filosofía y Sociopolítica de la Educación, 4(8), 13-28.

Escribano, O. (2008). El proceso de investigación social cualitativo. Buenos Aires, Argentina: Editorial prometeo.

Stake, R. (Ed.). (1999). Investigacion con estudio de casos. Ediciones Morata. Madrid, España

Tolozano Benites, S. E., Lara Díaz, L. M. y Illescas Prieto, S. (2016). Actitudes y aptitudes del tutor para enfrentar el desafío de la formación en la modalidad dual. Revista Universidad y Sociedad, 8, 81-91.

Tolozano, S. Lara, L. y Illesca, S. (2016). Actitudes y aptitudes del tutor para enfrentar el desafío de la formación en la modalidad dual Revista Científica de la Universidad de Cienfuegos, 8(1), 81-91.

Torrado-Arenas, D. Manrique-Hernández, E. y AyalaPimentel, J. (2016). La tutoría entre pares: una estrategia de enseñanza y aprendizaje de histología en la Universidad Industrial de Santander. Medicas UIS, 29, 71-75.

UACH. (2017). Manual metodologico. Universidad autonoma de Chile.

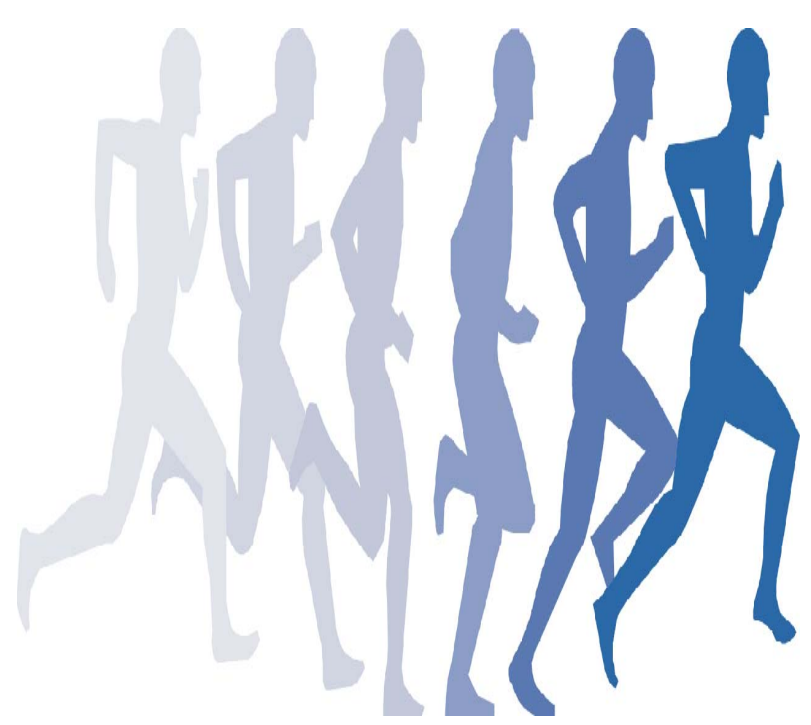

